\title{
Building a Trust Model for Social Network
}

\author{
Osama Rababah ${ }^{1} \&$ Bassam Alqudah $^{2}$ \\ ${ }^{1}$ King Abdullah II School of Information Technology, The University of Jordan, Amman, Jordan \\ ${ }^{2}$ School of Educational Sciences, The University of Jordan, Amman, Jordan \\ Correspondence: Osama Rababah, King Abdullah II School of Information Technology, Amman, Jordan. E-mail: \\ o.rababah@ju.edu.jo
}

Received: November 20, 2017

Accepted: February 28, 2018

Online Published: March 20, 2018

doi:10.5539/mas.v12n4p69

URL: https://doi.org/10.5539/mas.v12n4p69

\begin{abstract}
The revolution in information technology and the use of the internet changed the lifestyle of people. A major change was in the way of shopping. Companies started to offer their products online using social network s and people started to buy from the internet. Using social network has many benefits to the users starting from exploring a large variety of products to the very first way of ordering and the availability of the products 24 hours a day. One of the main problems that is found in using a social network is trusting the using social network social network s. the student concern about trusting to buy from the using social network social network s. Trust is a major concern for the merchant too; his concern is how to gain the student trust and to keep it. Many factors play a major role in acquiring the student trust in the online market. These factors rely on the social network characteristics such as design, interactivity and age and other factors vary from the social network quality, service quality, security policy of the social network, the privacy policy, the guarantee offered, the satisfaction of the user, the ease of use, the risk aversion and the culture factors. This study introduces the trusting affecting factors mentioned above and their effect on the trustworthiness factors (ability, benevolence, and integrity) a trust model has been built to show the relation between these factors and the trustworthiness factors.
\end{abstract}

Keywords: trust model, social trust, social networks, trust, trust affecting factors

\section{Introduction}

Recent years witnessed a huge revolution of using of social network all over the world; many students are adapting their study to the online environment and to take advantages of the social network in order to improve their studying and get new information. Joining social network social network s through the internet brought new information from different cultures and countries in comparing with official learning methods and social network s. It provides a wide range of pages and information to choose from that is easily accessed, However internet users still hesitate trust these social networks due to a lake of trust toward people who are joining the social network that they may be fake users (hudaib and fakhouri, 2018).

Previous researchers have shown that lack of trust constituted to the using of online social network social networks (Gefen and Straub, 2003; Caldwell \& Clapham 2003), determining the factors that increase trust in using social network plays an essential role in using social network successes.

this study proposes a trust model for social network by studying the effect of trust affecting factors that include Communication methods, Security policy, privacy policy, culture, Satisfaction, Social network Design, social network age, social network interactivity, Brand, Social network Quality, Service Quality and Ease of Use, content of social network, fast delivery . this research study the effect of each of these factors on the trustworthiness factors that have been proposed in the trust model of Caldwell \& Clapham 2003 which suggests that the trust have three trustworthiness factors: Ability, benevolence, and integrity.

The Internet has firmly entered into the life of modern youth. Today, many users pay great attention to popular social networks. Such social network s have many supporters, but also many opponents. Everyone, from which lead a lot of arguments in favor of their point of view. Especially the virtual world is at the moment attractive in the eyes of young people. In social networks, they can not only share their emotions and experiences, join groups of interests, but also have the opportunity to express themselves and get acquainted with new people, etc. It is also worth noting that an important feature of visiting young people's social communities is communication with friends, acquaintances, and relatives; acquaintance with their peers, the need for self-expression of self-identity. 
Users find here a moral outlet, closing themselves from the outside world. For example, currently in Jordan for 2017, the social network daily visits more than 3 million users, for social network s such as Facebook, Twitter, and Instagram.

There are not only advantages of social networks, there are also disadvantages. More often than not, the Internet community is replacing virtual communication with virtual ones. Many people even embellish their personal qualities during social networking. Man is closing himself to the problems of the outside world, immersed in the virtual world. This phenomenon is called "loneliness in the crowd." That is, a person without communicating with real people is constantly alone with the computer, which cannot but affect the psyche. He begins to give up his favorite hobbies, there are gaps in the university, a lot of free time is lost, which could be used for something more useful, for example, for sports, for live communication with friends and family. This entails dependence, which can have an impact on the psychological and physical health of young people. Under the influence of the environment, in the process of education, the young person's socialization takes place, and his worldview is formed. The world is increasingly influenced by the media, especially the Internet. The unformed worldview of a young man experiences two powerful streams of positive and negative information. A huge amount of video, music, films are uploaded daily to social networks, among which you can find rare and valuable samples for every taste. In addition, young people, who are engaged in various creative activities, are able to find a circle of people for their own interests with the goal of improving and developing their skills.

When Web-based social networks first appeared, they were mostly exploited by individual users to keep in touch with their friends and families (Mika, 2007). With their popularity and phenomenal growth, governments and commercial enterprises have also looked at exploiting their potential to deliver and improve services (Jaeger et al., 2007) (Zappen et al., 2008) (Borchorst et al., 2011). However, not all social networks are successful: indeed, many social networks disappear because they fail to attract enough members or because there are not enough interactions amongst members to retain people.

\subsection{Statement of the Problem}

Using social network from the internet face a major problem which is lack of trust, many factors affect this problem and may either cause to increase or decrease the trust.

This research studies the factors that affect trust in using a social network to find the best factors that affect the trust to build the better environment

The study problem can be summarized in the following questions:

- Which social network website should the student trust to use?

- What are the trust factors that the student should depend on when accessing and using social network website?

- What is the security level for social network websites?

- Does Trust also have a significant influence on attitude towards using the social network and getting information from it?

A very significant reason for why the Jordanian students hesitate the using of a social network is because of the basic lack of trust that may be found between the user and other users in the social network.

The major points in trust lack that help to raise the problem of trust are:

Many users have fake profiles on the social network with face identity or personality

The existence or lack of trust between the using social network parts is not a short-term problem but also a long-term border.

Using social network is different from traditional social communication skills, it is characterized by virtual community, uncertainty, anonymity, and lack of control and potential opportunism, in using social network the interaction will be mediated (e.g. by messenger, comment, like and posting media files and information) and many communication methods that are present in face-to-face encounters are not available or and don't exist virtual social network website.

Loss of communication and it is subject to hacking or fraud is often considered to increase uncertainty, and to result in lower level of trust. 


\subsection{Study objective}

In the guides of what has been discussed in the previous section, this study will try to answer the questions that have been raised in the Statement of the problem, and to achieve the following objectives:

1. To investigate the level of trust in using the social network in Jordan.

2. To investigate the factors that affect building the trust model in using the social network in Jordan.

3. To determine the degree of importance for each of the factors that affect trust in using the social network in Jordan.

4. To organize the findings in a descriptive trust model.

5. To make recommendations and suggestions for improvement in accordance with the study findings.

\subsection{Limitation of the Study}

The using social network world is incredibly dynamic in nature and today's experiences and perceptions maybe superseded by new technology. Therefore, the findings represent the current technological environment.

\section{Related Work}

Ankolekar et al., 2007 explored and analyzed whether trust, which is formally described as a"relationship in which a trustor decides to depend on the trustee's foreseeable behavior in order to fulfill his expectations", can be applied to illustrate a particular connection among users who interact exclusively online (M. Taddeo and L. Floridi, 2011).Cai-Nicolas Ziegler and Georg Lausen (2005) in their study First, introduced a classification scheme for trust metrics along various axes and discuss advantages and drawbacks of existing approaches for Semantic Web scenarios. Hereby, we devise an advocacy for local group trust metrics, guiding us to the second part which presents Appleseed, our novel proposal for local group trust computation. Compelling in its simplicity, Appleseed borrows many ideas from spreading activation models in psychology and relates their concepts to trust evaluation in an intuitive fashion.

Hee-Chul Choi DERI, et al,(2006) in their study detailed how trust can be modeled within online communities. We present methods for constructing community-aware identity management systems and for computing trust levels between users of a social network, using a novel trust model that takes advantage of both the capabilities of the Semantic Web and of a distributed topology. We also describe how the trust of a particular person relies on the separate social networks that they are members of. Finally, we evaluate our research against current studies in the psychology domain. Zhiyong Zhang and Kanliang Wang (2013), proposed a multimedia social networks trust model based on small world theory. By introducing some share character factors, such as credible feedback of digital contents, feedback weighting factor and, user share similarity, this model proposed a direct trust calculation window mechanism, recommended path finding algorithm, and multiple recommendation trust synthetic strategy.

Shree et al, (2014) studied several trust and reputation models and issues such as trust bootstrapping, trust evidence, trust assessment, second-order issues, interaction outcome evaluation, punishment, reputation propagation, redemption, context awareness, rewarding, dynamic nature and trust type value are being analyzed. Yang Wang and Alfred Kobsa (2009) conducted a study that aims at starting the groundwork towards filling the gap. Based on a review of existing literature in social networks and workplace studies, we hypothesize a number of potential privacy issues in this work practice and suggest future research directions in this area. Nepal et al., (2011) proposed a trust model for social networks with the aim of building trust communities that inspire members to share their experiences, feelings, and opinions in an open and honest way without the fear of being judged. The unique feature of our model is that the trust value is derived from the social capital built in the social networks over a period of time. Reyhan Aydo gan, et al., (2015) provided in their research a novel computational model for situation awareness of an intelligence agent has been proposed. The model is based on the theoretical three-level SA model of Endsley and Hoogendoorn's computational model of SA. The contribution of this work is threefold.

Jian-Ping Meia, et al, (2016) studied people's trust and rating behavior with the Epinions dataset. Epinions.com is a popular product review social network allowing users to rate various categories of products, and establish a list of trustworthy users. We perform the correlation analysis of activeness and trustworthiness defined by the number of ratings and the number of trustors to derive findings that can help the design of new decision support mechanisms in trust-based recommender systems. We then propose a trustee-influence based trust model where a trustee's activeness or trustworthiness is used to determine trust relationships. This trust model is incorporated into a memory-based and matrix factorization recommender systems to support online purchasing 
decision-making. Experimental results demonstrate the effectiveness of the proposed trust model for a recommendation.

W.Sherchan (2013) presented a comprehensive review of trust in social networks. We examined the definitions and measurements of trust through the prisms of sociology, psychology, and computer science. This study identified the various facets/aspects of trust as calculative, relational, emotional, cognitive, institutional/system, and dispositional. The study also identified the various properties of trust: context-dependent, dynamic, transitive, propagative, composable, personalized, asymmetric, self-reinforcing, and event-sensitive.

Iraklis Varlamis, et al, studied the contribution of various measures in identifying trustworthy or influential actors in a social network in order to recommend them to a septic user. Iraklis Varlamis, et al, Application of social network metrics to a trust-aware collaborative model for generating personalized user recommendations.M. Nick Hajli (2013) focused in their study on examining the role of social factors on trust, which can influence an individual's intention to buy. An understanding of this issue can aid in identifying factors that establish trust and social commerce intention through SNSs. M. Nick Hajli, A study of the impact of social media on consumers, International Journal of Market Research Vol.5 6 Issue 3,2013. Tom Heath, et al, have presented our approach to generating trust profiles for members of a user's social network, in the context of word of mouth recommendation seeking. This approach is based on algorithms for computing person-topic (expertise, experience) and person-person (affinity) trust metrics, that have been developed based on previous research. By utilizing people's social networks, and employing a rich model of trust in recommendation seeking, our approach overcomes the limitations of previous work in the field. Tom Heath,, et al, Computing Word-of-Mouth Trust Relationships in Social Networks for Semantic Web and Web2.0 Data Sources,. Krishnaprasad Thirunarayan, et al,. (2010) we developed a framework for describing the semantics of trust in social networks containing referral trust, (non)functional trust, trust scopes, etc., by exploiting and adapting many evidence-based insights. Meanwhile, Nahier Aldhafferi, et al, (2013) study aimed to measure the awareness of users on protecting their personal information privacy, as well as the suitability of the privacy systems which they use to modify privacy settings. A research conducted by Davis Bundi Ntwiga, et al, (2016) aimed to model trust of agents using the peer to peer reputation ratings in the network that forms a real-valued matrix.

James Caverlee, et al, (2008) proposed the Social Trust framework for tamper-resilient trust establishment in online social networks. Two of the salient features of Social Trust are its dynamic revision of trust by (i) distinguishing relationship quality from the trust; and (ii) incorporating a personalized feedback mechanism for adapting as the social network evolves.

Another research by Sa. Shekarpour, et al, introduced a Trust model for the semantic web. It is associated with a propagation method which computes trust through a chain of acquaintance and an aggregation method to select a path of the friend. Further, the method assigns each path a weight. These weights are assigned based on trust rating of friends and density. The trust rating of a tutor to a trustee in a path is selected from the path with the highest weight. The proposed method is implemented and its accuracy evaluated. Finally, the results obtained were compared with some techniques to calculate trust. Experimental results illustrate the effectiveness of the proposed method.

Whereas, Cai-Nicolas Ziegler and Georg Lausen in (2005) introduced a classification scheme for trust metrics along various axes and discuss advantages and drawbacks of existing approaches for Semantic Web scenarios. Hereby, we devise an advocacy for local group trust metrics, guiding us to the second part which presents Appleseed, our novel proposal for local group trust computation. Abedelaziz Mohaisen, et al, (2010) Designed Account for Trust in Social Network-based Sybil Defenses the designs are motivated by the observed relationship between the algorithmic property required for the defenses to perform well and a hypothesized trust value in the underlying graphs.

DuBois et al,., we present a new method for computing both trust and distrust (i.e., positive and negative trust). We do this by combining an inference algorithm that relies on a probabilistic interpretation of trust based on random graphs with a modified spring-embedding algorithm. Our algorithm correctly classifies hidden trust edges as positive or negative with high accuracy. These results are useful in a wide range of social web applications where trust is important to user behavior and satisfaction. Tomáš Knapand Irena Mlýnková, Towards, showed in the financial motivational scenario that the generic notion of trust is neither applicable nor reasonable. To that end, we proposed the novel topic-based trust model, and (1) introduced Topic-based Trust Module to persist the trusts model in a FOAF social network, (2) presented the TopicTrust algorithm (TTA) computing the topic-based trust among two persons in the FOAF social network by leveraging the algorithm TidalTrust with the vital concepts of topics, and (3) surveyed the topic hierarchies suitable for classifying the 
topics used in the trust model and suggested WordNet Domains as the topic hierarchy .

\section{Data Analysis}

The purpose of this section is to describe the analyses of the information and the data collected from the questionnaire. 420 questionnaires filled online were used for analysis adopting Statistical Package for Social Sciences (SPSS) software. The results were presented in two phases; the first one presents the descriptive data analysis. The second phase intended to present the results of the testing hypotheses of the research.

\subsection{Demographic Data Analysis}

The demographic items that were investigated in this research are gender, age, Education Level, university, the field of study, using the internet, using social network social network, Table 1 shows the Demographic data analysis.

Table 1. Demographic data analysis

\begin{tabular}{llll}
\hline Demographic Variables & & Frequency & percentage \\
\hline Gender & Male & 199 & $47.38 \%$ \\
& Female & 221 & $52.62 \%$ \\
Age & $18-21$ & 197 & $46.91 \%$ \\
& $22-25$ & 166 & $39.52 \%$ \\
\multirow{3}{*}{ Student status } & Over 26 & 57 & $13.57 \%$ \\
\multirow{2}{*}{ Using the internet/day } & Undergraduate student & 361 & $85.95 \%$ \\
& Graduate student & 59 & $14.05 \%$ \\
Using any of social network s per month & $1-3$ & 84 & $20.00 \%$ \\
& $3-6$ & 195 & $46.43 \%$ \\
& $6+$ & 141 & $33.57 \%$ \\
& $1-3$ & 52 & $12.38 \%$ \\
& $4-6$ & 73 & $17.38 \%$ \\
\hline
\end{tabular}

\subsubsection{Pearson Correlation Between Trustworthiness Factors}

By calculating the Pearson correlation between the three trustworthiness factors as shown in the following table using SPSS. it has been noticed that the correlation values are: $0.853,0.102,0.069$ respectively in since the Pearson Correlation between them are low then we will study the effect of each of the factors of the trust for integrity, ability, benevolence. Pearson Correlation between factors of the trust (ability, integrity, benevolence) is shown in table 2.

Table 2. Pearson Correlation between factors of the trust (ability, integrity, benevolence)

\begin{tabular}{lllll}
\hline & & ability & integrity & benevolence \\
\hline Ability & Pearson Correlation & 1 & .069 & $.853^{* *}$ \\
& Sig. (2-tailed) & & .102 & .000 \\
\multirow{5}{*}{ Integrity } & $\mathrm{N}$ & 566 & 566 & 566 \\
& Pearson Correlation & .069 & 1 & $.091^{*}$ \\
& Sig. (2-tailed) & .102 & & .030 \\
Benevolence & $\mathrm{N}$ & 566 & 566 & 566 \\
& Pearson Correlation & $.853^{* *}$ & $.091^{*}$ & 1 \\
& Sig. (2-tailed) & .000 & .030 & \\
& $\mathrm{~N}$ & 566 & 566 & 566 \\
\hline
\end{tabular}

**. Correlation is significant at the 0.01 level (2-tailed).

*. Correlation is significant at the 0.05 level (2-tailed). 
The total correlation between the three factors is:

Pearson Correlation of ability + Pearson Correlation of benevolence + Pearson Correlation of integrity $=0.853+$ $0.91+0.30=2.063$

\subsection{Hypotheses Testing}

Hypotheses testing involve testing the null hypotheses (denoted by H0) which are assumed to be true but tested for possible rejection. The probability value obtained from the statistical hypotheses test is considered decision rule for rejecting null hypotheses study (Creswell, 2003). If the probability value is less than or equal to a predetermined level of significance ( $\alpha$-level 0.05 ) the null hypotheses study will be rejected, and the alternative hypotheses study will be supported. Table 3 shows Hypotheses testing

Table 3. Hypotheses testing

\begin{tabular}{llll}
\hline Hypotheses & Ability & Benevolence & Integrity \\
\hline Social network framework Design & 0.574 & 0.701 & 0.129 \\
Social network framework interactivity & 0.569 & 0.799 & 0.024 \\
$\begin{array}{l}\text { Social network framework age } \\
\text { Social network framework quality }\end{array}$ & 0.31 & 0.294 & 0.307 \\
$\begin{array}{l}\text { Social network framework working } \\
\text { technically }\end{array}$ & 0.193 & 0.225 & 0.118 \\
Simple to navigate & 0.141 & 0.169 & 0.223 \\
Good Content social network & & & \\
Service Quality & 0.008 & -0.010 & 0.092 \\
Social network framework that & -0.007 & -0.021 & -0.035 \\
delivery & -0.005 & 0.012 & 0.023 \\
Communication methods & 0.003 & 0.032 & -0.061 \\
Security Policies & & & \\
Privacy Policies & 0.243 & 0.244 & 0.102 \\
Cultural Factors & 0.159 & 0.214 & 0.155 \\
Risk Aversion & 0.139 & 0.184 & 0.209 \\
Guarantees & 0.861 & 0.902 & 0.103 \\
Satisfaction & 0.863 & 0.984 & 0.091 \\
Brand & 0.589 & 0.692 & 0.112 \\
Ease of Use & 0.079 & 0.079 & 0.093 \\
\hline
\end{tabular}

\section{Hypotheses Study Description}

Linear regression Test was used for hypotheses study testing, using SPSS and by referring to the decision rule, for each null-hypotheses study it is either accepted or rejected. the partial relation value between the dependant variable ability and the studied factor indicated either it has a positive significant effect or don't in students' opinion on social network ability, benevolence and integrity trust factors, as shown in table 4, table 5 and table 6 .

Table 7. Factor Null Hypotheses study Acceptance / Rejection

\begin{tabular}{lll}
\hline Factor & Null Hypotheses study & $\begin{array}{l}\text { Acceptance/ } \\
\text { Rejection }\end{array}$
\end{tabular}

\footnotetext{
Social network H0.a: Social network framework design significantly does not have a Rejected framework Design positive impact on ability trust factor.
}

H0.b: Social network framework design significantly does not have a Rejected positive impact on benevolence trust factor.

H0.c: Social network framework design significantly does not have a Rejected positive impact on integrity trust factor. 


\begin{tabular}{|c|c|c|}
\hline $\begin{array}{l}\text { Social networ } \\
\text { framework }\end{array}$ & $\begin{array}{l}\text { H0.a: Social network framework interactivity significantly does not have a } \\
\text { positive impact on ability trust factor. }\end{array}$ & $d$ \\
\hline \multirow[t]{2}{*}{ interactivity } & $\begin{array}{l}\text { H0.b: Social network framework interactivity significantly does not have a } \\
\text { positive impact on benevolence trust factor. }\end{array}$ & Rejected \\
\hline & $\begin{array}{l}\text { interactivity significantly does not have } \\
\text { factor. }\end{array}$ & Rejected \\
\hline \multirow[t]{3}{*}{$\begin{array}{l}\text { Social network } \\
\text { framework age }\end{array}$} & $\begin{array}{l}\text { H0.a: Social network framework age significantly does not have a positive } \\
\text { impact on ability trust factor. }\end{array}$ & Rejected \\
\hline & $\begin{array}{l}\text { H0.b: Social network framework age significantly does not have a positive } \\
\text { impact on benevolence trust factor. }\end{array}$ & Rejected \\
\hline & ge significantly does not have a positive & Accepted \\
\hline \multirow[t]{3}{*}{$\begin{array}{l}\text { Social network } \\
\text { framework quality }\end{array}$} & $\begin{array}{l}\text { H0.a: Social network framework quality is negatively related to ability } \\
\text { trust factor. }\end{array}$ & Rejected \\
\hline & $\begin{array}{l}\text { H0.b: Social network framework quality is negatively related to } \\
\text { benevolence trust factor. }\end{array}$ & $\mathrm{Re}$ \\
\hline & $\begin{array}{l}\text { H0.c: Social network framework quality is negatively related to integrity } \\
\text { trust factor. }\end{array}$ & Rejected \\
\hline \multirow{3}{*}{$\begin{array}{l}\text { Social } \\
\text { framework } \\
\text { working } \\
\text { technically }\end{array}$} & $\begin{array}{l}\text { H0.a: Social network framework that works very well technically is } \\
\text { negatively related to ability trust factor. }\end{array}$ & Rejec \\
\hline & $\begin{array}{l}\text { H0.b: Social network framework that works very well technically is } \\
\text { negatively related to benevolence trust factor. }\end{array}$ & $\mathrm{Re}$ \\
\hline & $\begin{array}{l}\text { c: Social network framework that works very } \\
\text { gatively related to integrity trust factor. }\end{array}$ & $\mathrm{Re}$ \\
\hline
\end{tabular}

Simple to navigate

H0.a: Social network framework that is simple to navigate is negatively related to ability trust factor.

H0.b: Social network framework that is simple to navigate is negatively related to benevolence trust factor.

H0.c: Social network framework that is simple to navigate is negatively related to integrity trust factor.

Good Content
social network

Service Quality

Social network framework that has fast delivery

\section{Communication methods}

Security Policies
H0.a: Social network framework that has good content is negatively related to ability trust factor.

H0.b: Social network framework that has good content is negatively related to benevolence trust factor.

H0.c: Social network framework that has good content is negatively related to integrity trust factor.

H0.a: Service quality is negatively related to ability trust factor

H0.b: Service quality is negatively related to benevolence trust factor H0.c: Service quality is negatively related to integrity trust factor

H0.a: Social network framework that has fast delivery is negatively related to ability trust factor

H0.b: Social network framework that has fast delivery is negatively related to benevolence trust factor

H0.c: Social network framework that has fast delivery is negatively related to integrity trust factor

H0.a: Communication methods doesn't have a positive significant impact on ability trust factor

H0.b: Communication methods doesn't have a positive significant impact on benevolence trust factor

H0.c: Communication methods don't have positive significant effect on user trust on integrity trust factor.

H0.a: Security doesn't have positive significant effect towered ability trust Rejected factor

Accepted

Accepted

Rejected

Accepted

Accepted

Rejected

Accepted

Accepted

Accepted

Rejected

Rejected

Rejected
Accepted

Accepted

Rejected 
H0.b: Security doesn't have positive significant effect towered Rejected benevolence trust factor

H0.c: Security doesn't have positive significant effect towered integrity Rejected trust factor

Privacy Policies

Cultural Factors

Risk Aversion

Guarantees

Satisfaction

Ease of Use
H0.a: social network framework social network framework don't protect Rejected the user privacy and thus it has a negative effect on ability trust factor

H0.b: social network framework social network framework don't protect the user privacy and thus it has a negative impact on benevolence trust factor

H0.c: social network framework social network framework don't protect the user privacy and thus it has a negative impact on integrity trust factor H0.a: Culture difference is negatively related to ability trust factor.

H0.b: Culture difference is negatively related to benevolence trust factor. H0.c: Culture difference is negatively related to integrity trust factor.

H0.a: Risk aversion is negatively related to the ability trust factor. H0.b: Risk aversion is negatively related to the benevolence trust factor. H0.c: Risk aversion is negatively related to the integrity trust factor.

H0.9.a: Guaranty is negatively related to the ability trust factor. H0.9.b: Guaranty is negatively related to the benevolence trust factor. H0.9.c: Guaranty is negatively related to the integrity trust factor.

H0.a: Satisfaction is negatively related to the ability trust factor. H0.b: Satisfaction is negatively related to the benevolence trust factor. H0.c: Satisfaction is negatively related to the integrity trust factor. H0.a: Ease of Use is negatively related to the ability trust factor.

H0.b: Ease of Use is negatively related to the benevolence trust factor.

H0.c: Ease of Use is negatively related to the integrity trust factor.
Rejected

Rejected

Rejected

Rejected

Rejected

Rejected

Rejected

Rejected

Rejected

Rejected

Rejected

Accepted

Accepted

Accepted

Accepted

Accepted

Accepted

It can be seen from the results that the partial relation value between the dependent variable ability, integrity and benevolence trust factor and the Social network framework interactivity is $0.574,0.701,0.129$ respectively; this indicates that Social network framework design has a positive significant impact on ability, integrity, benevolence trust factor. Table 7 shows Factor Null Hypotheses study Acceptance / Rejection

The total Correlation between social network framework interactivity effect and the three trust factors is calculated by the flowing equation as shown in Figure 1:

Total Correlation between trustworthiness factors and web interactivity $=$ partial Correlation with ability + partial Correlation with benevolence + partial Correlation with integrity.

Total Correlation $=0.574+0.701+0.129=1.404$

It can be seen from the results that The partial relation value between the dependent variable ability, integrity and benevolence trust factor and the Social network framework interactivity is $0.569,0.799,0.024$ respectively; this indicates that Social network framework interactivity has a positive significant impact on ability, integrity, benevolence trust factor. The total Correlation between social network framework interactivity effect and the three trust factors is calculated by the flowing equation:

Total Correlation between trustworthiness factors and web interactivity $=$ partial Correlation with ability + partial Correlation with benevolence + partial Correlation with integrity.

Total Correlation $=0.569+0.799+0.024=1.392$

It can be seen from the results that the partial relation value between the dependent variable ability, integrity and benevolence trust factor and the Social network framework quality is $0.193,0.225,0.118$ respectively; this indicates that Social network framework quality has a positive significant impact on ability, integrity, 
benevolence trust factor.

The total Correlation between social network framework quality effect and the three trust factors is calculated by the flowing equation as shown in Figure 4:

Total Correlation between trustworthiness factors and Social network framework quality $=$ partial Correlation with ability + partial Correlation with benevolence + partial Correlation with integrity

Total Correlation $=0.193+0.225+0.118=0.536$

the results show that the partial relation value between the dependent variable ability, integrity and benevolence trust factor and the Social network framework age is 0.31, 0.294, 0.307 respectively; this indicates that Social network framework age has a positive significant impact on ability, integrity, benevolence trust factor.

It can be seen from the results that the partial relation value between the dependent variable ability, integrity and benevolence trust factor and the Social network framework that work very well technically is $0.141,0.169,0.223$ respectively; this indicates that Social network framework that works very well technically have a positive significant impact on ability, integrity, benevolence trust factor.

The total Correlation between Social network framework that works very well technically effect and the three trustworthiness factors (ability, benevolence, and integrity) is calculated by the flowing equation as shown in Figure 5:

Total Correlation between trustworthiness factors and Social network framework that work well technically $=$ partial Correlation with ability + partial Correlation with benevolence + partial Correlation with integrity

Total Correlation $=0.141+0.169+0.223=0.533$

It can be seen from the results that the partial relation value between the dependent variable ability, integrity and benevolence trust factor and the Social network framework that is simple to navigate is $0.008,-0.010,0.092$ respectively; this indicates that Social network framework that is simple to navigate have a positive significant impact on ability, integrity, benevolence trust factor.

The total Correlation between Social network framework that is simple to navigate effect and the three trust factors is calculated by the flowing equation as shown in figure 6 :

Total Correlation between trustworthiness factors and Social network framework that is simple to navigate $=$ partial Correlation with ability + partial Correlation with benevolence + partial Correlation with integrity.

Total Correlation $=0.008+-0.010+0.092=0.09$

It can be seen from the results that the partial relation value between the dependent variable ability, integrity and benevolence trust factor and the Social network framework that has good content is $-0.007,-0.021,-0.035$ respectively; this indicates that Social network framework that has good content has a positive significant impact on ability, integrity, benevolence trust factor.

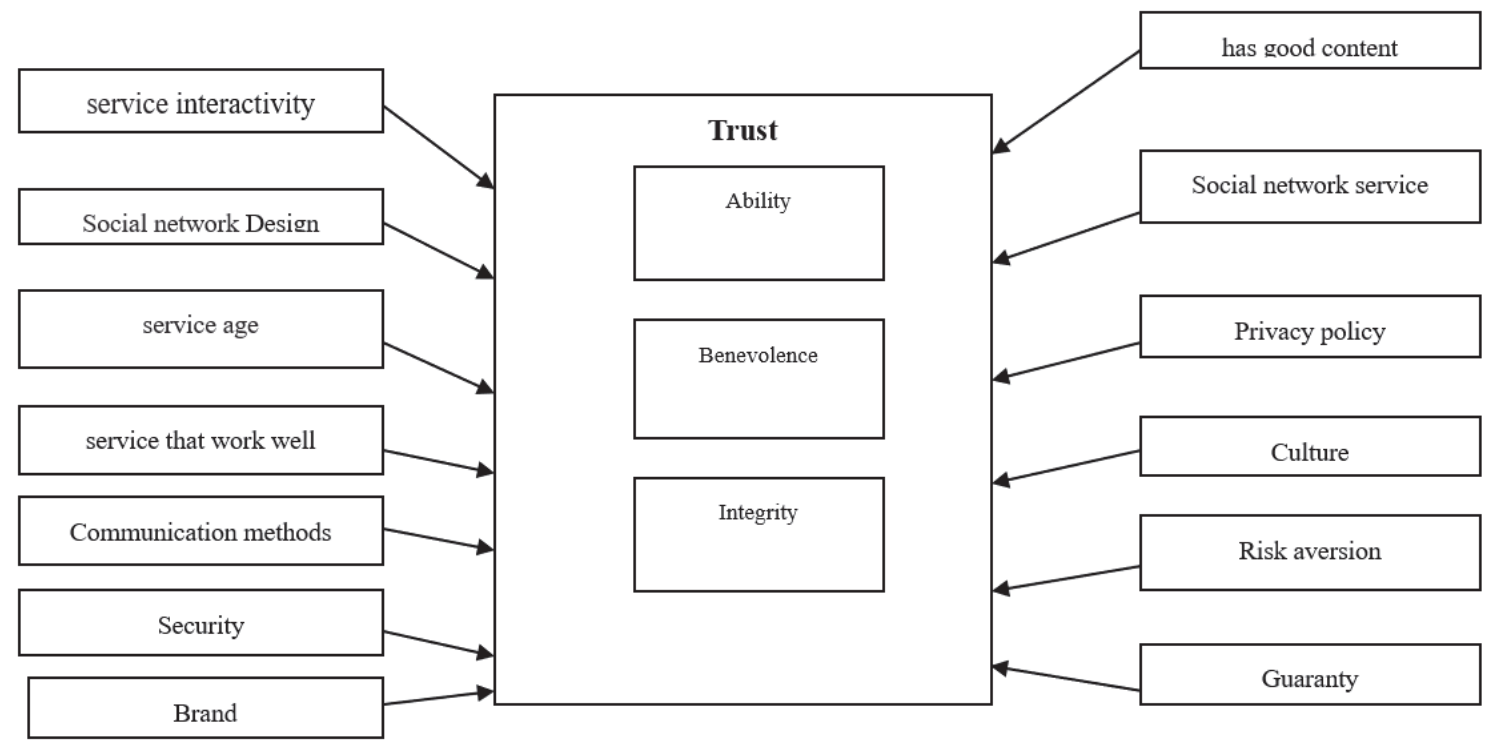

Figure 1. Trust model 
The total Correlation between Social network framework that has a good content effect and the three trust factors is calculated by the flowing equation:

Total Correlation between trustworthiness factors and Social network framework that has good content $=$ Partial Correlation with ability + partial Correlation with benevolence + partial Correlation with integrity.

Total Correlation $=-0.007+-0.021+-0.035=-0.063$

It can be seen from the results that the partial relation value between the dependent variable ability, integrity and benevolence trust factor and the Social network framework quality is $-0.005,0.012,0.023$ respectively; this indicates that Social network framework quality has a positive significant impact on ability, integrity, benevolence trust factor.

The total Correlation between Social network framework that has the good content effect and the three trust factors is calculated by the flowing equation as shown figure 8 :

Total Correlation between trustworthiness factors and Social network framework quality =

partial Correlation with ability + partial Correlation with benevolence + partial Correlation with integrity.

Total Correlation $=-0.005+0.012+0.023=0.030$

It can be seen from the results that the partial relation value between the dependent variable ability, integrity and benevolence trust factor and the Social network framework fast delivery is $0.003,0.032,-0.061$ respectively; this indicates that Social network framework fast delivery has a positive significant impact on ability, integrity, benevolence trust factor.

The total Correlation between Social network framework that has fast delivery and the trust factors is calculated by the flowing equation as shown figure 9 :

Total Correlation between trustworthiness factors and Social network framework that has fast delivery $=$

Partial Correlation with ability + partial Correlation with benevolence + partial Correlation with integrity.

Total Correlation $=0.003+0.032+-0.061=-0.026$

It can be seen from the results that the partial relation value between the dependent variable ability, integrity and benevolence trust factor and the Social network framework Communication methods is 0. 243, $0.244,0.102$ respectively; this indicates that Social network Communication methods have a positive significant impact on ability, integrity, benevolence trust factor.

The total Correlation between Communication methods and the trust factors is calculated by the flowing equation:

Total Correlation between trustworthiness factors and Communication methods $=$

Partial Correlation with ability + partial Correlation with benevolence + partial Correlation with integrity.

Total Correlation $=0.243+0.244+0.102=0.589$

It can be seen from the results that the partial relation value between the dependent variable ability, integrity and benevolence trust factor and the Social network framework Security is $0.159,0.214,0.155$ respectively; this indicates that Social network framework Security has a positive significant impact on ability, integrity, benevolence trust factor.

Total Correlation between trustworthiness factors and security $=$ partial Correlation with ability + partial Correlation with benevolence + partial Correlation with integrity

Total Correlation $=0.159+0.214+0.155=0.528$

It can be seen from the results that the partial relation value between the dependent variable ability, integrity and benevolence trust factor and the Social network framework Privacy policies is $0.139,0.184,0.209$ respectively; this indicates that Social network framework Privacy policies have a positive significant impact on ability, integrity, benevolence trust factor, as shown figure 13.

Total Correlation between trustworthiness factors and privacy $=$ partial Correlation with ability + partial Correlation with benevolence + partial Correlation with integrity

Total Correlation $=0.139+0.184+0.209=0.532$

It can be seen from the results that the partial relation value between the dependent variable ability, integrity and benevolence trust factor and the Social network framework Culture is $0.861,0.902,0.103$ respectively; this 
indicates that Social network framework Culture have a positive significant impact on ability, integrity, benevolence trust factor.

The total Correlation between culture effect and the three trust factors is calculated by the flowing equation:

Total Correlation between trustworthiness factors and culture $=$ partial Correlation with ability + partial Correlation with benevolence + partial Correlation with integrity

Total Correlation $=0.861+0.902+0.103=1.866$

It can be seen from the results that the partial relation value between the dependent variable ability, integrity and benevolence trust factor and the Social network framework Risk aversion is $0.863,0.984,0.091$ respectively; this indicates that Social network framework Risk aversion has a positive significant impact on ability, integrity, benevolence trust factor.

The total Correlation between risk aversion effect and the three trust factors is calculated by the flowing equation as shown figure 15:

Total Correlation between trustworthiness factors and risk aversion $=$ partial Correlation with ability + partial Correlation with benevolence + partial Correlation with integrity

Total Correlation $=0.863+0.984+0.091=1.941$

It can be seen from the results that the partial relation value between the dependent variable ability, integrity and benevolence trust factor and the Social network framework Guaranty is 0.598, 0.692, 0.112 respectively; this indicates that Social network framework Guaranty has a positive significant impact on ability, integrity, benevolence trust factor.

The total Correlation between guaranty effect and the three trust factors is calculated by the flowing equation,

Total Correlation between trustworthiness factors and guaranty $=$ partial Correlation with ability + partial Correlation with benevolence + partial Correlation with integrity.

Total Correlation $=0.589+0.692+0.112=1.393$

It can be seen from the results that the partial relation value between the dependent variable ability, integrity and benevolence trust factor and the Social network framework Satisfaction is 0.079, 0.079, 0.093 respectively; this indicates that Social network framework Satisfaction has a positive significant impact on ability, integrity, benevolence trust factor.

The total Correlation between satisfaction effect and the three trust factors is calculated by the flowing equation:

Total Correlation between trustworthiness factors and satisfaction $=$ partial Correlation with ability + partial Correlation with benevolence + partial Correlation with integrity

Total Correlation $=0.079+0.079+0.093=0.251$

It can be seen from the results that the partial relation value between the dependent variable ability, integrity and benevolence trust factor and the Social network framework Brand is 0.891, 0.993, 0.096 respectively; this indicates that Social network framework Brand has a positive significant impact on ability, integrity, benevolence trust factor.

The total Correlation between brand effect and the three trustworthiness factors (ability, benevolence, and integrity) is calculated by the flowing equation as shown figure 18 :

Total Correlation between trustworthiness factors and brand $=$ partial Correlation with ability + partial Correlation with benevolence + partial Correlation with integrity

Total Correlation $=0.891+0.993+0.096=1.980$

It can be seen from the results that the partial relation value between the dependent variable ability, integrity and benevolence trust factor and the Social network framework Ease of use is - 0.017, -0.034, 0.003 respectively; this indicates that Social network framework Ease of use has a positive significant impact on ability, integrity, benevolence trust factor.

The total Correlation between the ease of use and the three trustworthiness factors (ability, benevolence, and integrity) is calculated by the flowing equation as shown figure 19:

Total Correlation between trustworthiness factors and ease of use $=$ partial Correlation with ability + partial Correlation with benevolence + partial Correlation with integrity

Total Correlation $=-0.017+-0.034+0.003=-0.048$ 


\section{Conclusions and Recommendations}

this paper reflect the relationship between trust affecting factors like social network design, age, interactive, social network quality, service quality, fast delivery, available products, simple to navigate social network, security policy of the social network, privacy policy, the guarantee offered, the satisfaction of the user, the ease of use, the risk aversion and the culture factors on trustworthiness factors (ability, benevolence, and integrity).

the population of this study consists of undergraduates and graduate students from all schools of the University of Jordan with ages between 18 - 30 years old. Results also shown that they are using Facebook heavily given that $38.5 \%$ of students are spending three hours daily, According to data analysis it is found that the following factors positively affect the trust worthiness factor(ability, benevolence and integrity) in the case study of using social network in Jordan ( social network design, social network interactivity, social network age, social network quality, social network that work very well technically, communication methods, security policy, privacy policy, culture, risk aversion, guarantee, brand).A summary of the values for the octal correlation for positive trust affecting factors on trustworthiness are summarized in table 8 .

Table 8. Values for the octal correlation for positive trust affecting factors on a trustworthiness

\begin{tabular}{lll}
\hline no & Trust affecting factors & Total correlation \\
\hline $\mathbf{1}$ & social network design & 1.401 \\
$\mathbf{2}$ & social network interactivity & 1.378 \\
$\mathbf{3}$ & social network age & 0.873 \\
$\mathbf{4}$ & social network quality & 0.529 \\
$\mathbf{5}$ & a social network that works very well technically & 0.529 \\
$\mathbf{7}$ & communication methods & 0.574 \\
$\mathbf{8}$ & security policy & 0.521 \\
$\mathbf{9}$ & privacy policy & 0.532 \\
$\mathbf{1 0}$ & Culture & 1.855 \\
$\mathbf{1 1}$ & risk aversion & 1.935 \\
$\mathbf{1 2}$ & Guarantee & 1.385 \\
$\mathbf{1 3}$ & Brand & 1.965 \\
\hline
\end{tabular}

According to our data analysis, we found that the following factors don't have to affect the trustworthiness factors (ability, benevolence, and integrity) in the case study of using social network in Jordan (simple to navigate social network, social network content information, service quality, fast delivery, satisfaction, ease of use).A summary of the values of the total correlation for negative trust affecting factors on trustworthiness is summarized in table 9.

Table 9. Values of the total correlation for negative trust affecting factors on the trustworthiness

\begin{tabular}{lll}
\hline no & Trust affecting factors & Total correlation \\
\hline $\mathbf{1}$ & simple to navigate social network & 0.09 \\
$\mathbf{3}$ & service quality & 0.031 \\
$\mathbf{4}$ & fast delivery & 0.018 \\
$\mathbf{5}$ & Satisfaction & 0.254 \\
$\mathbf{6}$ & ease of use & -0.55 \\
\hline
\end{tabular}

This paper presented a comprehensive trust model in a social network. We examined the definitions and measurements of trust in social media. Many factors affect the problem of lack of trust and may either cause to increase or decrease the trust. This research studies the factors that affect trust in using social network finding the best factors that affect the trust to build a better environment. We describe the analyses of the information and the data collected from the questionnaire. The results were presented in two phases; the first one presents the descriptive data analysis. The second phase presents the results of the testing hypotheses of the research.

The recommendation of this research is first the social network in Jordan should take care of the positive affecting factors to build a trust level with the students. Second Jordan should care about training the people who work in the developing of a social network to be high professional about all the above-mentioned factors such as 
web design and quality in order to develop a trustable social network that could compete globally. The third recommendation using social network skills should be being listed as a major course in our Jordanian universities especially for students whom major are not related to information technology.

In Jordan, according to the data analysis provided in this research the following factors: Social network design, social network interactivity, social network age, social network quality, social network that work very well technically, communication methods, security policy, privacy policy, culture, risk aversion, guarantee, and brand, Positively affect the trustworthiness factor (ability, benevolence and integrity, and the following factors: simple to navigate social network, social network content information, service quality, fast delivery, satisfaction, and ease of use, don't have to affect the trustworthiness factors (ability, benevolence and integrity).

\section{References}

Abedelaziz Mohaisen, et al, Designs to Account for Trust in Social Network-based Sybil Defenses, Copyright is held by the author/owner(s). CCS'10, October 4-8, 2010, Chicago, Illinois, USA. ACM 978-1-4503-0244-9/10/10.

Amjad, A., \& Hudaib, H. N. (2018). Supernova Optimizer: A Novel Natural Inspired Meta-Heuristic. Modern Applied Science, 12(1). ISSN 1913-1844 E-ISSN 1913-1852, Published by Canadian Center of Science and Education. https://doi.org/10.5539/mas.v12n1p32

Amjad, A., Hudaib, H. N. F., Fatima, E. A. A., \& Sandi, N. F. (2017). A Survey about Self-Healing Systems (Desktop and Web Application. Scientific Research Publishing.

Ankolekar, M., Anupriya, T. T., \& Vrandecic, D. (2007). The two cultures: mashing up web 2.0 and the semantic web," in Proceedings of the 16th international conference on World Wide Web, WWW '07, (New York, NY, USA), pp. 825-834, ACM, 2007.

Basim, A., \& Hussam, N. F. (2008). Automation of iron deficiency anemia blue and red cell number calculating by intestinal villi tissue slide images enhancing and processing, Computer Science, and Information Technology, 2008. ICCSIT'08. International Conference.

Basim, A., Hussam, N. F., \& Omar, S. A. (2006). cDNA microarray genome image processing using fixed spot position. American Journal of Applied Sciences, 3(2), 1730-1734.

Borchorst, N. G., Bødker, S., Colineau, N., Korn, M. \& Paris, C. (eds.) (2011). Proceedings of the C\&T 2011 Workshop on Government and Citizen Engagement, Brisbane: IISI - International Institute for Socio-Informatics.

Cai-Nicolas, Z., \& Georg, L. (2005). Propagation Models for Trust and Distrust in Social Networks. Information Systems Frontiers, 7(4/5), 337-358. Springer Science + Business Media, Inc. Manufactured in The Netherlands

Cai-Nicolas, Z., \& Georg, L. (2005). Propagation Models for Trust and Distrust in Social Networks. Information Systems Frontiers, 7(4/5), 337-358. Springer Science + Business Media, Inc. Manufactured in The Netherlands.

Davis, B. N. et al. (2016). Trust Model for a social network using singular value decomposition. Interdisciplinary Description of Complex Systems, 14(3), 296-302.

Hee-Chul, C. D. et al. (2006). Trust Models for Community-Aware Identity Management. Copyright is held by the author/owner(s). 2006, May 22-26, 2006, Edinburgh, UK.

Jaeger, P. T., Shneiderman, B., Fleischmann, K. R., Preece, J., Qu, Y., \& Wu, P. F. (2007). Community response grids: E-government, social networks, and effective emergency management. Telecommunications Policy, 31, 592-604.

James, C. et al. (2008). Towards Robust Trust Establishment in Web-Based Social Networks with SocialTrust. April 21-25, 2008, Beijing, China.

Jian-Ping, M. et al. (2016). A Social Influence based Trust Model for Recommender Systems, Preprint submitted to Intelligent Data Analysis April 7, 2016.

Krishnaprasad, T. et al. (2010). Some Trust Issues in Social Networks and Sensor Networks,978-1-4244-6622-1/10 2010 IEEE.

Mika, P. (2007). Social Networks and the Semantic Web New York, Springer.

Nahier, A. et al. (2013). Personal information privacy settings of online social networks and their suitability for 
mobile internet devives. International Journal of Security, Privacy and Trust Management (IJSPTM), 2(2), April 2013.

Omar, A., Amjad, H., Hussam, N., \& Fakhouri, L. A. (2013). Microarray Gene Expression Extraction and Archiving Based on Local Spot Locations, IJCSI Int. J. Comput. Sci.

Reyhan, A. et al. (2015). A Trust-Based Situation Awareness Model, Springer International Publishing Switzerland $2015 \quad$ N. Bulling (Ed.): EUMAS 2014, LNAI 8953, 19-34. https://doi.org/10.1007/978-3-319-17130-22

Rizik, M. H., Al-Sayyed, H. N., Fakhouri, A. R., \& Colin, P. (2017). Polar Particle Swarm Algorithm for Solving Cloud Data Migration Optimization Problem. Modern Applied Science, 11(8), 98.

Shekarpour, S. et al. (2011). A Trust Model For Semantic Web, ISSN:1473-80x online, 1473-8031 print. IJSSST, $10(2)$.

Surya, N., Wanita, S., \& Cecile, P. S. (2011). Trust: A Trust Model for Social Networks. International Joint Conference of IEEE TrustCom-11/IEEE ICESS-11/FCST-11

Taddeo, M., \& Floridi, L. (2011). The case for e-trust. Ethics and Information Technology, 13, 1-3. https://doi.org/10.1007/s10676-010-9263-1.

Thomas, D. B., Jennifer, G., \& Aravind, S. (2013). Predicting Trust and Distrust in Social Networks.

Udhaya, S. S., \& Basha, M. S. S. (2014). An Exhaustive Survey of Trust Models in P2P Network. International Journal on Web Service Computing (IJWSC), 5(3).

W.Sherchan, A. (2013). Survey of Trust in Social Networks. ACM Computing Surveys, 45(4), 47, Publication date: August 2013.

Yang, W., \& Alfred, K. (2009). Privacy in Online Social Networking at Workplace, IEEE Int'l Conference on Computational Science and Engineering, Vancouver, Canada, pp. 975-978. https://doi.org/10.1109/CSE.2009.438

Zappen, J. P., Harrison, T. M., \& Watson, D. (2008). A new paradigm for designing e-government: web 2.0 and experience design. Proceedings of the 2008 international conference on Digital government research. Montreal, Canada: Digital Government Society of North America.

Zhang, Z. Y., \& Wang, K. L. (2013). A trust model for multimedia social networks. Soc. Netw. Anal. Min., 3, 969-979. https://doi.org/10.1007/s13278-012-0078-4

\section{Copyrights}

Copyright for this article is retained by the author(s), with first publication rights granted to the journal.

This is an open-access article distributed under the terms and conditions of the Creative Commons Attribution license (http://creativecommons.org/licenses/by/4.0/). 